\title{
1 Wild-captive contrasts in non-vocal communicative repertoires and functional specificity in 2 orang-utans
}

4 Marlen Fröhlich ${ }^{1 *}$, Natasha Bartolotta ${ }^{1}$, Caroline Fryns ${ }^{1}$, Colin Wagner $^{2}$, Laurene Momon ${ }^{2}$, Marvin

5 Jaffrezic $^{2}$, Tatang Mitra Setia ${ }^{3}$, Caroline Schuppli $^{1.4}$, Maria van Noordwijk ${ }^{1}$, Carel P. van Schaik ${ }^{1}$

6

$7 \quad{ }^{1}$ Department of Anthropology, University of Zurich, Zurich, Switzerland

$8{ }^{2}$ DEPE-IPHC - Département Ecologie, Physiologie et Ethologie, University of Strasbourg,

9 Strasbourg, France

$10 \quad{ }^{3}$ Fakultas Biologi, Universitas Nasional, Jakarta Selatan, Indonesia

$11{ }^{4}$ Leipzig Center for Early Child Development, Leipzig, Germany

12 *Corresponding author, email address: marlen.froehlich@uzh.ch

\section{Abstract}

15 The creation of novel communicative acts is an essential element of human language. Although some

16 research suggests the presence of this ability in great apes, this claim remains controversial. Here, we

17 use orang-utans (Pongo spp.) to systematically assess the effect of the wild-captive contrast on the

18 repertoire size of communicative acts. We find that individual communicative repertoires are

19 significantly larger in captive compared to wild settings, irrespective of species, age-sex class or

20 sampling effort. Twenty percent of the orang-utan repertoire in captivity were not observed in the

21 wild. In Sumatran orang-utans, the more sociable species, functional specificity was also higher in

22 captive versus wild settings. We thus conclude that orang-utans, when exposed to a more sociable 
23 and terrestrial lifestyle, have the behavioural plasticity to invent new communicative behaviours that

24 are highly functionally specific. This productive capacity by great apes is a major prerequisite for the

25 evolution of language and seems to be ancestral in the hominid lineage.

\section{Keywords}

28 Close-range communication, gesture, signal repertoire, signal meaning, behavioural plasticity, socio29 ecological environment, language evolution, Pongo abelii, Pongo pygmaeus

\section{Introduction}

32 One of the core features of human language is its productivity, referring to the idea that signallers

33 can create and understand novel utterances with novel meanings ${ }^{1}$. While several other building

34 blocks of language, including intentionality, reference and compositional syntax, have increasingly

35 been documented among a wide range of non-human species ${ }^{2,3,4}$, productivity seems to be very rare

36 in the animal kingdom. Instead, animal communication systems are thought to rely heavily on

37 evolved signals, that is, communicative acts whose form and function evolved under the influence of

38 the effect on the recipient ${ }^{5,6}$. The current consensus is that all facial, vocal and most gestural

39 expressions of non-human species have evolved through natural selection over long periods of time,

40 and have become innate: the ability to produce them arises spontaneously during ontogeny, whereas

41 its use is often fine-tuned by practice ${ }^{7,8,9}$.

43 Recent research on great apes, our closest living relatives, suggests that it may be timely to

44 distinguish between innate animal signals and those that are acquired developmentally ${ }^{10}$. Great apes

45 have provided most comparative evidence for the cognitive building blocks and selective pressures 
46 shaping the human communication system ${ }^{11,12,13}$. But recent work has also shown that some of their

47 gestures and sounds are apparently innovated and maintained over time ${ }^{14,15,16,17,18,19,20}$, and play

48 the same role in the communication process as evolved signals do - we could thus call them invented

49 signals. Because targeted studies to estimate the extent of productivity in great apes are so far

50 lacking, we here examine this question by comparing the same species in the wild and in a novel

51 setting, captivity ${ }^{10,21}$. The contrast allows a direct test of how repertoires respond to the changes in

52 the socio-ecological environment. In captivity, individuals face less competition for food, have more

53 spare time, are closer together, are more often visible to each other, and more on the ground than in

54 the wild ${ }^{22,23}$. Especially in fission-fusion species such as orang-utans (Pongo spp.), interaction rates

55 in contexts such as social play, grooming, conflict situations and mating are boosted in captive

56 settings e.g. ${ }^{24,25,26}$, which may favour the production of innovative communicative acts, and thus

57 cause differences in the communicative repertoires of individuals and groups.

59 The captive-wild contrast also allows us to examine the extent to which the meaning of

60 communicative acts depends on the context in which they are used. We predict that the learned,

61 "species-atypical" communicative acts have high functional specificity, i.e. a highly context-specific

62 production $^{10,27,28}$, a phenomenon reflecting communicative plasticity. This is because the use of

63 invented signals inevitably implies intentionality: naïve individuals observed and learned them,

64 inferred their meaning from context and reactions, and subsequently used them in the same context

65 with the same function as their original inventor but see 29,30 for contrary views on highly conserved signal production.

66 In contrast, evolved signals may be used intentionally or non-intentionally. They may therefore vary

67 in functional specificity, and show more communicative flexibility: the same communicative act

68 serves several different goals or functions, relying on context to provide disambiguation ${ }^{2,31}$. 
70 The aim of the present study was to examine repertoires and functional specificity of close-range

71 communicative acts, in both wild and captive populations of orang-utans, the great ape genus which

72 is in our view ideal for this avenue of research. Systematic studies on the gestural repertoire of

73 captive orang-utans have demonstrated that their propensity for elaborate and flexible gesture use

74 parallels that of other great apes ${ }^{32,33,34}$. This suggests that social propensities can be fully expressed

75 in captivity, as individuals do not need to be solitary in order to obtain sufficient food, whereas in the

76 wild they may have fewer interaction opportunities and communication is hampered by arboreality

77 and obscuring vegetation. In addition, mothers are the predominant communication partner of infant

78 orang-utans in the wild ${ }^{35,36}$, so that there is a limited need for the production of extensive

79 communicative repertoires. There are no systematic wild-captive comparisons of apes'

80 communicative behaviour to date, but we assume that contrasts must be larger for orang-utans than

81 any other great ape taxon.

We examined non-vocal (i.e. gestural and facial) communicative acts of Bornean and Sumatran orang-utans (Pongo pygmaeus/abelii) in two wild populations and five zoos. In a first step, we established the repertoires and functions (presumed goals of communicative acts, with outcomes that apparently satisfied the signaller) of orang-utans' non-vocal communicative acts, building on

87 previous work conducted on chimpanzees and captive orang-utans ${ }^{33,37}$, but separately for wild and

88 captive settings. We then tested several predictions about how setting affected individual repertoire

89 sizes and functional specificity of signal types, while controlling for important confounding variables

90 such as age-sex class and sampling effort. First, captivity should result in larger communicative

91 repertoires because of boosted territoriality, sociability and interaction rates. As a result, wild

92 repertoires should be a subset of the captive ones, except for those communicative acts that cannot be

93 expressed in captivity ("wild-only"). Second, we expect that the form of these communicative acts

94 expressed only in captivity should be tightly linked to the more terrestrial lifestyle or the increased 
95 sociability, especially in the less terrestrial Sumatran orang-utans. Third, the "invention" of

96 additional signals in captivity should be accompanied by a wild-captive contrast in functional

97 specificity. We expect to find this contrast especially or even exclusively in Sumatran orang-utans,

98 because sociability and interaction rates are reportedly higher in the Northwest-Sumatran population

99 compared to the Bornean populations ${ }^{38,39}$. The effect of this setting-species interaction on functional

100 specificity will allow us to derive important conclusions on the plasticity and flexibility underlying

101 communicative interaction in the Pongo genus.

103 Results

104 Communicative acts across settings

105 A total of 40 distinct signal types were identified across all settings, out of which 34 were observed

106 in Bornean (captive: $\mathrm{N}=27$, wild: $\mathrm{N}=24$ ) and 39 in Sumatran orang-utans (captive: $\mathrm{N}=37$, wild: $\mathrm{N}$

$107=32$ ). Plotting the cumulative number of identified communicative acts over the course of the

108 observation period indicated that study groups have been sufficiently sampled to grasp complete

109 repertoires (Fig. 1, S1, S2, S3), except for two captive groups of Bornean orang-utans (Apenheul and

110 Cologne, see Fig. S1). In Table S1 we provide definitions for all coded behaviours and their relation

111 to previous work on orang-utans' communicative repertoire. The majority of signal types (N) and

112 cases $(\mathrm{n})$ consisted of manual $(\mathrm{N}=19, \mathrm{n}=5106)$ and bodily signals $(\mathrm{N}=18, \mathrm{n}=2212)$, whereas

113 considerably fewer facial acts $(\mathrm{N}=3, \mathrm{n}=110)$ act were observed (see Tab. S2 for detailed overview

114 of signal presence in relation to settings, species, subjects and age classes). The relatively small

115 repertoire of facial signals may be partly due to our strict criteria of inclusion into the repertoire (see 116 methods). 
118 We first tested the first prediction that captivity should result in enlarged communicative repertoires

119 at the aggregate level owing to boosted sociability and interaction rates. We found that the majority

120 of communicative acts $(\mathrm{N}=27)$ was shared across orang-utan species and research settings, but that

1219 communicative acts were restricted to captivity (e.g. "roll on back", "throw object", "somersault",

122 see Fig. 2), and 2 to the wild ("loud scratch", "shake object", see Fig. 2), thus confirming the

123 prediction. Out of these, seven captivity-specific and one wild-specific acts were observed in the

124 Sumatran species only (e.g. "rub on body", "head-butt", "shake object"), whereas one behaviour

125 ("spin") was observed in captive Borneans only (see Tab. S2 for a detailed overview communicative

126 acts across settings and species). A more conservative way of testing the prediction is by producing a

127 list based on all previous studies. We found that three of the communicative acts we found only in

128 captive settings ("throw object", "rise up") or wild settings ("shake object"), were also observed in

129 other species-setting combinations in other studies, which leaves at least seven captivity-only acts

130 and one wild-only act (see Tab. S3, note that Cartmill \& Byrne [2010] do not specify which gestures

131 were observed in which orang-utan species). This more conservative test thus also confirms the

132 prediction.

134 To ensure that these differences between captivity and the wild do not reflect differences in social

135 opportunities (e.g. with regard to the availability same-age play partners), we compiled separate play

136 repertoires for mother-offspring (for which there is no change in partner availability between natural

137 and captive settings) versus same-aged interactions (Tab. S4). A graphical analysis revealed no

138 substantial difference between captive versus wild play repertoires with regard to mother-offspring

139 interactions (25 vs 24 in Sumatrans, 22 vs 18 in Borneans). For peer play interactions, repertoire

140 sizes apparently differed between settings for Sumatrans (26 vs. 19), but not Borneans (12 vs. 11).

141 Differences in repertoire sizes between captive and wild settings are thus not driven by partner

142 availability alone. Given that 13 communicative acts used in same-aged play interactions were 
143 exclusively used in Sumatrans (Tab. S4), we suggest that repertoire size is driven more by social

144 opportunities in Sumatrans than in Borneans.

\section{Individual repertoire sizes across settings}

147 The average Bornean orang-utans had 1.9 more signal types in its repertoire (captive: mean $\pm \mathrm{SD}=$ 148 8.1 \pm 3.8 , wild: $10 \pm 4.7$ ), while the average Sumatran had 6.8 more signal types (captive: mean \pm

$149 \mathrm{SD}=15.3 \pm 6.5$, wild: $8.5 \pm 7.2$ ), with a larger between-individual variation. With regard to age 150 classes, we found that younger immatures had the largest repertoire on average (mean $\pm \mathrm{SD}=14.8 \pm$ $1515.9, \mathrm{~N}=42$ ), followed by older immatures $(10.1 \pm 8, \mathrm{~N}=41)$, and adults (mean $\pm \mathrm{SD}=7.3 \pm 3.9, \mathrm{~N}$ $152=37)$.

Using a linear mixed model (LMM), we tested how setting, species and confounding variables such

155 as sex, age class and sampling effort affected the number of communicative acts in individuals

156 recorded during the study (for details see methods). The full model including the key test predictors

157 (i.e. setting and species) fitted the data better than the null models irrespective of the subsets used

158 (LRT all individuals: $\chi^{2}{ }_{3}=14.058, P=0.003, N=70$; individuals with $>50$ interactions: $\chi^{2}{ }_{3}=$

$15915.131, P=0.002, N=44)$. As expected, the number of communicative acts were strongly affected

160 by the number of samples contributed to the dataset (see Tab. 1 for output of the model using the

161 restricted dataset, Tab. S5 for the model including all individuals). Irrespective of the effects of

162 sampling effort, however, we found that captive individuals deployed a significantly larger variety of

163 communicative acts than their wild counterparts, again confirming our prediction. We also found that

164 individual repertoires of Sumatran individuals exceeded those of their Bornean counterparts (after

165 removing the non-significant interaction term, Tab. 1 a, Fig. 3 a, b). In addition, younger individuals

166 produced significantly more different communicative acts than adults (Tab. 1 a, Fig. S4). These 
167 results are consistent with our expectations that young individuals (i.e. all immatures, but especially

168 those below the age of 5 years) regularly use a larger communicative set than adults, particularly for

169 the function of soliciting social play and food sharing. This is further supported by descriptive results

170 on presumed goals and outcomes of communicative acts (see ESM, Tab. S6, S7) suggesting that

171 interactions in both affiliative and conflict situations rather than co-locomotion or food-sharing

172 underlie the proliferation of communicative acts in captive settings.

\section{Functional specificity of communicative acts across settings}

175 We systematically tested the second prediction on functional specificity of communicative acts

176 depends on research setting and orang-utan species using a LMM, which also included confounding

177 variables such as outcome and sampling effort. The full model including the key test predictors (i.e.

178 setting, species, dominant outcome) fitted the data better than the null models (LRT: $\chi^{2}{ }_{3}=22.612, P$

$179<0.001, N=114)$. There was a significant interaction between research setting and orang-utan

180 species: while specificity scores in captivity did not differ between the species, we found a

181 significantly lower functional specificity in wild Sumatrans compared to their captive counterparts

182 (Fig. 4). Irrespective of this result, specificity was significantly higher for communicative acts

183 predominantly used to solicit play and observed in a larger number of subjects (Tab. 1 b). Our

184 findings thus support the prediction that larger repertoire sizes in captivity should be accompanied by

185 an increase in average functional specificity.

\section{Discussion}

Answering the question whether our primate relatives possess the behavioural plasticity, or creative

capacity, to complement their species-typical repertoires by inventing novel signals from scratch is 
191 examined communication systems of apes exposed to novel socioecological conditions relative to the 192 wild baseline situation. Here, we adopted a 2 × 2 comparative design, investigating repertoire sizes

193

194 and functional specificity of communicative acts in zoo-housed and wild groups of two different orang-utan species with different sociability and terrestriality. By examining the captive-wild contrast in these two related species we tested the prediction that captive environments favour the emergence of novel communicative acts, which should also have higher functional specificity.

Moreover, comparing species differences related to differential sociability and terrestriality on one hand, and setting on the other, we expected that Sumatran orang-utans, but not Borneans, would show a wild-captive contrast in average functional specificity, offering insights into the degrees of plasticity and flexibility underlying the communicative repertoires of orang-utans.

Consistent with our first prediction, communicative repertoires on both the aggregate and individual level were larger in captivity as compared to the wild, even after controlling for the expected effects of age class and sampling effort (i.e. irrespective of whether all or only highly sampled individuals are included in the analysis). There may be some doubt that a single study can exhaustively sample signal repertoires. We therefore also compared the captive-wild contrast for each species using all available studies. This comparison supported the conclusions based on our study, in that the actual repertoire composition found in previous studies in the same setting and species revealed no major differences (Tab. S2, S3). First, our own findings regarding zoo repertoires (i.e. 27 different communicative acts in captive Borneans and 37 in captive Sumatrans, as compared to 24 and 32 communicative acts in the wild, respectively) are broadly consistent with the available systematic studies in single settings. Liebal and colleagues ${ }^{32}$, studying two captive groups of Sumatran orangutans, reported a repertoire of 34 signal types (29 gestures, and 5 facial expressions). Like them, we found that the majority of communicative acts were used to solicit social play and food transfers. Cartmill \& Byrne ${ }^{33}$, examining two zoo groups of Bornean and one group of Sumatran orang-utans, 
216 identified 38 types of gesture and facial expressions that allowed the analysis of "intentional

217 meaning”. Second, the first systematic study on mother-offspring gesture use among wild orang-

218 utans, conducted at the Bornean population of Sabangau Forest, identified 21 gesture types that met

219 the criteria for inclusion into the repertoire ${ }^{35}$. With 24 different observed communicative acts

220 observed in our study population at Tuanan, it seems like the inclusion of communicative

221 interactions outside the mother-offspring bond does not result in a substantially larger repertoire size.

222 Thus, we can conclude that moving wild orang-utans into captivity leads to a 20 to $25 \%$ increase (i.e.

2237 to 9 acts "gained", 1 to 2 acts "lost") in their repertoire of communicative acts.

225 The second prediction we made was that the form of these communicative acts expressed only in 226 captivity should be tightly linked to the increased sociability and more terrestrial lifestyle. As

227 expected, differences in repertoire size were particularly pronounced for presumed goals related to 228 seeking body contact ("Play/affiliate", "Groom", "Sexual contact") and social conflict ("Move 229 away" and "Stop action"). Interaction rates with these outcomes are greatly boosted in captivity, 230 where a more differentiated use of bodily communication is both enabled and required ${ }^{10}$. Captive 231 facilities are stable, plentiful and predator-free environments that may provide opportunities for, and 232 even require (e.g. due to increasing conflict with limited space) signal inventions and innovations, 233 just like they foster innovations in general ${ }^{40,41}$. Our findings thus provide direct evidence that the 234 new environments we have created for great apes boost the invention of new signals, which may 235 spread through social learning. Indeed, captive settings in general have generated extensive and 236 convincing evidence for invented ("species-atypical") signalling, encompassing novel pant-hoot 237 variants ${ }^{42}$, and "whistling" 15 , as well as pointing with hands and fingers ${ }^{43,44}$, "raspberries" and 238 "extended grunts" in chimpanzees ${ }^{16}$. Although detailed captive-wild comparisons are, to our 239 knowledge, so far lacking for other great apes, wild-captive contrasts are probably larger in orang- 
240 utans than any other great ape taxon. In conclusion, our results support the notion that the new

241 opportunities and needs linked to captivity may lead to a proliferation of signal invention.

243 We also expected that the form of these communicative acts expressed only in captivity are linked to 244 a more terrestrial lifestyle. Indeed, we found that the communicative acts that are exclusively (or 245 overwhelmingly) produced in captive settings are strongly linked to the more terrestrial nature of 246 their artificial habitat: "somersaults", "spitting", "head-stands", communicative acts that involve 247 either the ground or objects obtained from the ground, would be very difficult to perform by wild 248 orang-utans with their purely (Sumatra) or predominantly Borneo: ${ }^{45}$ arboreal lifestyle. This setting

249 effect is not attributable to the presence of certain interaction partners alone: by comparing the 250 repertoires for mother-offspring and peer play interactions we demonstrated that differences between 251 interaction dyads with regard to wild-captive contrasts were only strong in Sumatran orang-utans. It 252 thus appears that the new affordances of captive settings, on top of the elevated exposure to certain 253 social contexts, enabled orang-utans to better exploit their (communicative) motion spectrum, 254 resulting in novel communicative movements that may independently and predictably such as 255 spitting as an attention-getter, see ${ }^{46}$ be invented in several captive colonies and species. This also 256 confirms earlier reports making the case that the complex individual-based fission fusion structure of 257 orang-utans and their sophisticated social-cognitive skills seem to be reflected in a highly variable 258 communicative repertoire ${ }^{32,47}$, illustrating their remarkable behavioural plasticity.

260 Finally, we predicted that the additional signals in captivity should be accompanied by an increase in 261 functional specificity. In line with our predictions, we found that Sumatrans in the wild exhibited a 262 lower average functional specificity compared to their captive counterparts, while average functional 263 specificity in captivity did not differ between the species. When comparing Sumatrans' 
264 communicative acts used only in captivity ("invented signals") with those used in both research

265 settings, captivity-only acts appeared to be on average more functionally specific (although the small

266 sample prevented inferential analyses). In other words, wild Sumatrans seem to use their

267 communicative acts more flexibly (i.e. redundantly) across presumed goals than in captivity, which

268 appears to be largely due to captive Sumatrans' use of invented context-dependent, and therefore

269 functionally specific acts (i.e. those that have "tight meanings" according to ${ }^{33}$ ) not present in the

270 wild repertoire. In contrast, the relatively low interactions rates (due to few social opportunities) of

271 wild Bornean orang-utans ${ }^{38,39,48}$ seem to be reflected in a lesser need to use signals flexibly across

272 contexts. Together, these results corroborate our expectation that average functional specificity

273 increased with repertoire size in captive Sumatran, though not Bornean orang-utans.

275 To convey a message that can be understood by a targeted recipient, an intentional agent may use

276 two alternative "strategies". First, she can use one and the same communicative act for several

277 different functions, relying on context or other information (e.g. possibly age difference or sex

278 relative to recipient) to disambiguate between ambiguous meanings. This "flexible" communicative

279 strategy produces redundancy in the communicative repertoire. Alternatively, she can use a

280 communicative behaviour that is specific to one interaction outcome - and, if not available, invent

281 one for which naïve recipients (over repeated instances of interaction) infer their meaning from

282 context and reactions, which they may subsequently use in exactly the same contexts with exactly the

283 same function as their original inventor. This "plastic" communicative strategy produces productivity

284 in the communicative repertoire. Sumatran orang-utans in the wild seem to rely somewhat more on

285 the flexibility option; their captive counterparts, however, rely more on the plasticity option because

286 repeated interactions with the same partners in captivity allow them to establish novel signal

287 meanings more rapidly. This outcome is expected because novel signals are most likely to be

288 understood and thus maintained when they are highly context-specific. 
290 We argue that distinguishing species-typical from invented communicative acts matters greatly for

291 current debates on language origins see also ${ }^{10}$. Language reflects extreme communicative plasticity.

292 Words that make up language are predominantly invented noises used intentionally, and the

293 productivity feature of language fundamentally relies on the ability to produce such new noises,

294 which are used intentionally and rapidly acquire a shared meaning due to their high context-

295 specificity. Functionally specific signals are often more effective and efficient in complex

296 interactional exchanges (including language), because they are less dependent on context and thus

297 less ambiguous. Reliance on context may be less favourable with increasing message complexity

298 (multiple intertwining messages), because it increases both (i) the risk of misunderstanding, and (ii)

299 the "decoding work" necessary for the recipient, and thus the risk that the signal is ignored.

Although our knowledge of the taxonomic distribution of invented signals is still incomplete, they are so far reported almost exclusively for great apes. Ongoing work supports this pattern, in that great apes are increasingly documented to make up new vocal e.g. ${ }^{15,16,49}$ and gestural e.g. ${ }^{18,50}$ 'signals' in the novel conditions of captivity, whereas reports from other taxa are rare but see e.g. ${ }^{51}$ $52,53,54$. Such communicative creativity may therefore be most common in, or even limited to, species with intentional communication. These findings imply that once the conditions were in place that

307 favoured the open-ended use of invented expressions, our hominin ancestors readily responded to 308 this opportunity, because they could build on a long evolutionary history of communicative

309 creativity. This might explain why language evolved in the hominin lineage and not others that found 310 themselves in similar conditions (e.g. those canids that also rely on interdependent foraging and 311 cooperative breeding). 


\section{Materials and Methods}

314

\section{Data collection}

Data were collected at two field sites and five captive facilities (zoos). We observed wild orang-utans at the long-term research sites of Suaq Balimbing (0302’ N; 97²5’E, Gunung Leuser National Park,

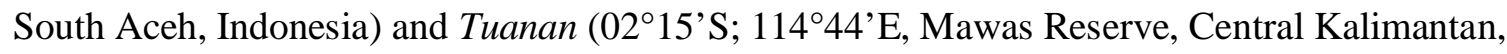
Indonesia), on a population of wild Sumatran (Pongo abelii) and Bornean orang-utans (Pongo pygmaeus wurmbii), respectively. Both study sites consist mainly of peat swamp forest and show high orang-utan densities, with 7 individuals per $\mathrm{km}^{2}$ at Suaq and 4 at Tuanan ${ }^{55,56}$. Captive Bornean orang-utans were observed at the zoos of Cologne and Münster, and at Apenheul (Apeldoorn), while Sumatran orang-utans were observed at the zoo of Zurich and at Hellabrunn (Munich; see EEP studbook for details on captive groups; Becker 2016). While captive Sumatran orang-utans were housed in groups of nine individuals each, captive Bornean groups were smaller (on average four individuals, with the one in Apenheul including only a mother and her dependent and independent offspring). Signallers included in this study consisted of 33 Bornean (21 wild/12 captive) and 38 Sumatran orang-utans (20 wild/18 captive; see Tab. S8 for detailed information on subjects and group compositions).

Focal observations were conducted between November 2017 and October 2018 (Suaq Balimbing: November 2017 - October 2018; Tuanan: January 2018 - July 2018, European zoos: January 2018 June 2018). At the two field sites, these observations consisted of full (nest-to-nest) or partial follows (e.g. nest-to-lost or found-to-nest) of mother-infant units, whereas in zoos 6-hour focal follows were conducted. Two different behavioural sampling methods were combined: First, presumable intraspecific communicative interactions of all observed social interactions of the focal either as signaller or receiver with all partners $(N=7137$ acts), and among other conspecifics ( $N=888$ acts $)$ present 
were recorded using a digital High-Definition camera (Panasonic HC-VXF 999 or Canon Legria HF M41) with an external directional microphone (Sennheiser MKE600 or ME66/K6), enabling recordings of high-quality footage. In captive settings with glass barriers, we also used a Zoom H1 Handy recorder that was placed in background areas of the enclosure whenever possible. Second, using instantaneous scan sampling at ten-minute intervals, we recorded complementary data on the activity of the focal individual, the distance and identity of all association partners, and in case of social interactions the interaction partner as well as several other parameters. During ca. 1600 hours of focal observations, we video-recorded more than 6300 communicative interactions.

\section{Coding procedure}

A total of 2655 high-quality recordings of orang-utan interactions (wild: 1643, captive: 1012) were coded using the program BORIS version 7.0.4. ${ }^{58}$. We designed a coding scheme to enable the analysis of presumably communicative acts directed at conspecifics (i.e. behaviours that apparently served to elicit a behavioural change in the recipient and were mechanically ineffective i.e. achieve a presumed goal without physical force; see also ${ }^{33}$. Manual, bodily, and facial acts were defined and aligned (see Tab. S1) based on previous studies on orang-utan communication in captive $26,32,33,46$ and wild settings ${ }^{35,36,59,60}$. Comparing our dataset to this literature, we then identified the subset of setting- and species-specific communicative acts. Although we also coded vocalizations based on field studies ${ }^{61}$, we did not include vocalizations in the analyses of repertoire and functional specificity as we could not equally pick up soft, low-frequency sounds in captive and wild settings, which hampered the fine-grained comparison across settings. For each communicative act, we coded the following "modifiers": presumed goal following the distinction of ${ }^{33}$, outcome, and other variables not directly relevant in this study (see Tab. S9 for levels and definitions of all coded variables). To ensure inter-observer reliability, we evaluated the coding performance of all observers 
361 with alternating datasets using the Cohen's Kappa coefficient ${ }^{62}$ during an initial training period and

362 at regular intervals afterwards. Trained observers (MF, NB, CF, CW, LM, MJ) proceeded with video

363 coding only if at least a 'good' level $(\kappa \geq 0.75)$ of agreement was found for signal type, articulator,

364 sensory modality, context, and response. For our repertoire analyses, we plotted the cumulative

365 number of communicative behaviours over the number of coded interactions for each study group

366 (Fig. S1, S2) and for a subset of highly sampled individuals (Fig. S3), to estimate how many

367 observations are necessary to grasp the repertoire of these groups/individuals as indicated by an

368 asymptote. Communicative acts were counted as part of individuals' repertoire only when observed

369 at least twice per subject.

371 To analyse functional specificity, we focused on goal-outcome matches ${ }^{33}$ or apparently satisfactory

372 outcomes ${ }^{37}$, that is, whether the interaction outcome aligned with presumed goals identified by

373 observers. We considered only those signal types that were produced at least three times towards a

374 particular interaction outcome cf. ${ }^{37}$. We defined functional specificity depending on how often a

375 communicative act was produced towards an apparently satisfactory outcome (ASO), adopting the

376 definitions of Cartmill and Byrne ${ }^{33}$. Signal types that were used mainly towards a single interaction

377 outcome, i.e. more than $70 \%$ of the time, were defined as having "tight meanings". Signal types used

378 frequently towards a single ASO, i.e. 50-70\% of the time, were defined as having "loose meanings".

379 Finally, signal types that were used less than $50 \%$ of the time towards a single ASO were classified

380 as having "ambiguous meanings". For instance, "somersault" was exclusively produced to initiate

381 "Play/affiliate" interactions (specificity value of 1, tight meaning), whereas "touch" was produced

382 towards several different interaction outcomes, e.g. "Play/affiliate", "Share food/object" and "Co-

383 locomote" (specificity values $<0.7$, ambiguous or loose meaning). 


\section{Statistical analyses}

386 We ran two separate linear mixed models LMMs; ${ }^{63}$ with a Gaussian error structure and identity link 387 function to examine sources of variation in (a) individual communicative repertoires (i.e. number of 388 signal types used at least twice) and (b) specificity in signal function. We used LMMs rather than 389 GLMMs in this study because it has recently been shown that linear models are more robust to 390 violations of distributional assumptions ${ }^{64,65}$. We ran model (a) for two subsets of our data: first, 391 including all individuals regardless of sample size; second, including only those individuals that 392 contributed more than 50 communicative acts to the dataset (as the graphical inspection of asymptote 393 plots in Fig. S3 suggested that this is a representative number to estimate individual repertoire sizes).

In model (a), which had individual repertoire size as response variable, we included research setting

396 (2 levels: captive, wild) and orang-utan species (2 levels: Bornean, Sumatran) as our key test

397 predictors. Because we assumed that the effect of research setting might depend on genetic

398 predisposition (i.e. species), we included the interaction between these two variables into our model.

399 Moreover, immature individuals often contribute the majority of both signal cases and types ${ }^{2,}$ for

400 reviews see ${ }^{31}$ and the composition of study groups differed with regard to age-sex classes, hence we 401 made sure that age class was taken into account in our analyses. We included the following variables 402 as additional fixed effects (control predictors) into the models: subjects' age class (3 levels: "adult":

403 females > 15 years, males > 16 years; "older immature": independent and dependent immature > 5

404 years of age, "younger immature": dependent immature < 5 years of age), sex (2 levels: female,

405 male), the number of interaction outcomes the subject communicated for at least twice (range $=1-6$,

406 only four outcomes were coded in captive Borneans), and the number of observations (range = 5-

407 467). To control for repeated measurements within the same sampling unit, group identity was 
408 treated as random effect. To keep type 1 error rates at the nominal level of 5\%, we also included all

409 relevant random slopes components within group ID ${ }^{66}$.

411 In model (b), which had specificity in signal function as response variable, we included orang-utan

412 species (two levels: Bornean, Sumatran), research setting (two levels: captive, wild) and dominant

413 outcome (two levels: play, non-play), and the interaction between setting and species as our key test

414 predictors. To control for confounding effects of sampling effort, the number of subjects contributing

415 to the use of a signal type (range $=1-21$ ) and the number of observations (range $=1-787$ ) in the

416 respective setting as additional fixed effects (i.e. control predictors). To control for repeated

417 observations of the same signal types across settings, signal type was treated as random effect ${ }^{67}$.

419 All models were implemented in R $\left(\mathrm{v} 3.4 .1,{ }^{68}\right)$ using the function glmer of the package lme $4{ }^{69}$. To

420 control for collinearity, we determined the Variance Inflation Factors VIF; ${ }^{70,71}$ from a model

421 including only the fixed main effects using the function vif of the R package car ${ }^{72}$. This revealed no

422 collinearity issues (maximum VIF $=2.8$ ). Prior to running the models, we log-transformed the

423 response variables and the control variables relating to sampling effort (number of

424 observations/subjects), to achieve an approximately symmetrical distribution and avoid influential

425 cases.). To test the overall significance of our key test predictors ${ }^{73,74}$, we compared the full models

426 with the respective null models comprising only the control predictors as well as all random effects

427 using a likelihood ratio test ${ }^{75}$. Tests of the individual fixed effects were derived using likelihood

428 ratio tests (R function dropl with argument "test" set to "Chisq"). 
431 General: We thank Erin Vogel and Suci Utami Atmoko (Tuanan), Kerstin Bartesch (Tierpark

432 Hellabrunn), Claudia Rudolf von Rohr (Zoo Zürich), Alexander Sliwa (Kölner Zoo), Simone Sheka

433 (Allwetterzoo Münster) and Thomas Bionda (Apenheul Primate Park) as well as all research staff

434 and zoo keepers for a fruitful collaboration during this study. We gratefully acknowledge Clemens

435 Becker for providing the EEP studbook, the Indonesian State Ministry for Research and Technology

436 (RISTEK), the Indonesian Institute of Science (LIPI), the Directorate General of Natural Resources

437 and Ecosystem Conservation - Ministry of Environment \& Forestry of Indonesia (KSDAE-KLHK),

438 the Ministry of Internal affairs, the Nature Conservation Agency of Central Kalimantan (BKSDA),

439 the local governments in Central Kalimantan, the Kapuas Protection Forest Management Unit

440 (KPHL), the Bornean Orang-utan Survival Foundation (BOSF) and MAWAS in Palangkaraya.

441 Moreover, we thank Simone Pika and Eva Luef for providing some of the essential equipment,

442 Santhosh Totagera for coding assistance, Uli Knief and Alex Hausmann for help with a customized

443 jitter-plot function. Funding: MF was supported by the Deutsche Forschungsgemeinschaft (DFG,

444 German Research Foundation, DFG, grant no- FR 3986/1-1) the Forschungskredit Postdoc (grant

445 FK-17-106) and the A.H. Schultz Foundation of the University of Zurich, the Sponsorship Society of

446 the German Primate Center (DPZ), the Stiftung Mensch und Tier (Freiburg) and the Christiane

447 Nüsslein-Volhard Foundation. CvS acknowledges the support of the NCCR Evolving Language

448 Program (SNF \#51NF40_180888). Author contributions: MF and CvS conceived of the study. MF

449 designed the project, collected, coded and analysed data. NB, CF, CW, LM, MJ helped to collect,

450 curate and code data. TMS, CS, MvN and CvS provided resources. MF wrote the manuscript with

451 inputs from CF, LM, CS, MvN and CvS. All authors approved the submission of the manuscript.

452 Competing interests: The authors declare no competing interests. Data and materials

453 availability: All data needed to evaluate the conclusions in the paper and the R code can be found on

454 GitHub: https://github.com/MarlenF/repertoire-orang. 


\section{References}

457 1. Hockett CF. The origin of speech. Scientific American 203, 88-97 (1960).

458

459

460

461

462

463

464

465

466

467

468

469

470

471

472

473

474

475

476

477

478

479

480

481

482

483

484

485

486

487

488

489

490

491

492

493

494
2. Liebal K, Waller BM, Burrows AM, Slocombe KE. Primate Communication: A Multimodal Approach. Cambridge University Press (2013).

3. Townsend SW, et al. Exorcising Grice's ghost: an empirical approach to studying intentional communication in animals. Biol Rev 92, 1427-1433 (2017).

4. Sievers C, Gruber T. Reference in human and non-human primate communication: What does it take to refer? Anim Cogn 19, 759-768 (2016).

5. Maynard Smith J, Harper D. Animal signals. Oxford University Press (2003).

6. Bradbury JW, Vehrencamp SL. Principles of animal communication. Sinauer (1998).

7. Cheney DL, Seyfarth RM. Flexible usage and social function in primate vocalizations. Proc Natl Acad Sci USA 115, 1974-1979 (2018).

8. Wegdell F, Hammerschmidt K, Fischer J. Conserved alarm calls but rapid auditory learning in monkey responses to novel flying objects. Nature Ecology \& Evolution 3, 1039-1042 (2019).

9. Fröhlich M, Hobaiter C. The development of gestural communication in great apes. Behav Ecol Sociobiol 72, 194 (2018).

10. Fröhlich M, van Schaik CP. Must all signals be evolved? A proposal for a new classification of communicative acts. Wiley Interdisciplinary Reviews: Cognitive Science 11, e1527 (2020).

11. Tomasello M. Origins of human communication. MIT press (2008).

12. Levinson SC, Holler J. The origin of human multi-modal communication. Philosophical Transactions of the Royal Society of London B: Biological Sciences 369, 20130302 (2014).

13. Fröhlich M, Sievers C, Townsend SW, Gruber T, van Schaik CP. Multimodal communication and language origins: integrating gestures and vocalizations. Biol Rev 94, 1809-1829 (2019).

14. Wich SA, et al. Call cultures in orang-utans? PLoS ONE 7, e36180 (2012). 
495 15. Wich SA, Swartz KB, Hardus ME, Lameira AR, Stromberg E, Shumaker RW. A case of spontaneous acquisition of a human sound by an orangutan. Primates 50, 56-64 (2009).

497

498

499

500

501

502

503

504

505

506

507

508

509

510

511

512

513

514

515

516

517

518

519

520

521

522

523

524

525

526

527

528

529

530

531

532

533

534

535

536

16. Hopkins WD, Taglialatela JD, Leavens D. Chimpanzees differentially produce novel vocalizations to capture the attention of a human. Anim Behav 73, 281-286 (2007).

17. Hardus ME, Lameira AR, Van Schaik CP, Wich SA. Tool use in wild orang-utans modifies sound production: a functionally deceptive innovation? Proc R Soc Lond, Ser B: Biol Sci 276, 3689-3694 (2009).

18. Halina M, Rossano F, Tomasello M. The ontogenetic ritualization of bonobo gestures. Anim Cogn 16, 653-666 (2013).

19. Fröhlich M, Wittig RM, Pika S. Should I stay or should I go? Initiation of joint travel in mother-infant dyads of two chimpanzee communities in the wild. Anim Cogn 19, 483-500 (2016).

20. Tomasello M, Call J, Warren J, Frost GT, Carpenter M, Nagell K. The ontogeny of chimpanzee gestural signals: A comparison across groups and generations. Evolution of Communication 1, 223-259 (1997).

21. Kummer H, Goodall J. Conditions of innovative behaviour in primates. Philos Trans R Soc Lond, Ser B: Biol Sci 308, 203-214 (1985).

22. Fröhlich M, van Schaik CP. The function of primate multimodal communication. Anim Cogn 21, 619-629 (2018).

23. Partan SR, Marler P. Communication goes multimodal. Science 283, 1272-1273 (1999).

24. Kopp KS, Liebal K. Conflict resolution in socially housed Sumatran orangutans (Pongo abelii). PeerJ 6, e5303 (2018).

25. Maple TL. Orangutan behavior. Van Nostrand Reinhold Co. (1980).

26. Zucker EL, Mitchell G, Maple T. Adult male-offspring play interactions within a captive group of orang-utans (Pongo pygmaeus). Primates 19, 379-384 (1978).

27. Brainard MS, Doupe AJ. What songbirds teach us about learning. Nature 417, 351-358 (2002).

28. Beecher MD, Brenowitz EA. Functional aspects of song learning in songbirds. Trends in Ecology \& Evolution 20, 143-149 (2005). 
540

541

542

543

544

545

546

547

548

549

550

551

552

553

554

555

556

557

558

559

560

561

562

563

564

565

566

567

568

569

570

571

572

573

574

575

576

577

578

579

29. Byrne RW, Cartmill E, Genty E, Graham KE, Hobaiter C, Tanner J. Great ape gestures: intentional communication with a rich set of innate signals. Anim Cogn 20, 755-769 (2017).

30. Fischer J, Hammerschmidt K. Towards a new taxonomy of primate vocal production learning. Philosophical Transactions of the Royal Society B: Biological Sciences 375, 20190045 (2020).

31. Call J, Tomasello M. The gestural communication of apes and monkeys. Lawrence Erlbaum Associates (2007).

32. Liebal K, Pika S, Tomasello M. Gestural communication of orangutans (Pongo pygmaeus). Gesture 6, 1-38 (2006).

33. Cartmill E, Byrne R. Semantics of primate gestures: intentional meanings of orangutan gestures. Anim Cogn 13, 793-804 (2010).

34. Cartmill EA, Byrne RW. Orangutans modify their gestural signaling according to their audience's comprehension. Curr Biol 17, 1345-1348 (2007).

35. Knox A, et al. Gesture use in communication between mothers and offspring in wild orangutans (Pongo pygmaeus wurmbii) from the Sabangau Peat-Swamp Forest, Borneo.

International Journal of Primatology 40, 393-416 (2019).

36. Fröhlich M, Lee K, Mitra Setia T, Schuppli C, van Schaik CP. The loud scratch: a newly identified gesture of Sumatran orangutan mothers in the wild. Biol Lett 15, 20190209 (2019).

37. Hobaiter C, Byrne Richard W. The meanings of chimpanzee gestures. Curr Biol 24, 15961600 (2014).

38. van Schaik CP. The socioecology of fission-fusion sociality in orangutans. Primates 40, 6986 (1999).

39. Fröhlich M, et al. Social interactions and interaction partners in infant orang-utans of two wild populations. Anim Behav 166, 183-191 (2020).

40. van Schaik CP. The primate origins of human nature. ( $\mathrm{ed}^{\wedge}(\mathrm{eds})$. John Wiley \& Sons (2016).

41. Lehner SR, Burkart JM, van Schaik CP. An evaluation of the geographic method for recognizing innovations in nature, using zoo orangutans. Primates 51, 101-118 (2010).

42. Marshall AJ, Wrangham RW, Arcadi AC. Does learning affect the structure of vocalizations in chimpanzees? Anim Behav 58, 825-830 (1999). 
580

581

582

583

584

585

586

587

588

589

590

591

592

593

594

595

596

597

598

599

600

601

602

603

604

605

606

607

608

609

610

611

612

613

614

615

616

617

618

619

620

621

622

43. Leavens DA, Hopkins WD. Intentional communication by chimpanzees: A cross-sectional study of the use of referential gestures. Dev Psych 34, 813-822 (1998).

44. Leavens DA, Russell J, Hopkins W. Multimodal communication by captive chimpanzees (Pan troglodytes). Anim Cogn 13, 33-40 (2010).

45. Ashbury AM, et al. Why do orangutans leave the trees? Terrestrial behavior among wild Bornean orangutans (Pongo pygmaeus wurmbii) at Tuanan, Central Kalimantan. Am J Primatol 77, 1216-1229 (2015).

46. Jantschke F. Orang-Utans in Zoologischen Gärten. Piper (1972).

47. Maestripieri D. Primate social organization, gestural repertoire size, and communication dynamics. In: The Origins of Language: What Nonhuman Primates Can Tell ( $\mathrm{ed}^{\wedge}(\mathrm{eds}$ King BJ). School of American Research Press (1999).

48. Mitra Setia T, Delgado R, Utami Atmoko S, Singleton I, van Schaik CP. Social organization and male-female relationships. In: Orangutans: Geographic variation in behavioral ecology and conservation $\left(\mathrm{ed}^{\wedge}(\right.$ eds Wich SA, Utami Atmoko S, Mitra Setia T, van Schaik CP). Oxford University Press (2009).

49. Taglialatela JP, Reamer L, Schapiro SJ, Hopkins WD. Social learning of a communicative signal in captive chimpanzees. Biol Lett 8, 498-501 (2012).

50. Tomasello M, Call J, Nagell K, Olguin R, Carpenter M. The learning and use of gestural signals by young chimpanzees: A trans-generational study. Primates 35, 137-154 (1994).

51. Moura ACdA. Stone banging by wild capuchin monkeys: an unusual auditory display. Folia Primatol 78, 36-45 (2007).

52. Garland Ellen C, et al. Dynamic Horizontal Cultural Transmission of Humpback Whale Song at the Ocean Basin Scale. Curr Biol 21, 687-691 (2011).

53. Grant BR, Grant PR. Cultural inheritance of song and its role in the evolution of Darwin's finches. Evolution 50, 2471-2487 (1996).

54. Perry S, et al. Traditions in wild white-faced capuchin monkeys. In: The biology of traditions: Models and evidence (ed^(eds Fragaszy DM, Perry S). Cambbridge University Press (2003).

55. Singleton I, Knott C, Morrogh-Bernard H, Wich S, van Schaik C. Ranging behavior of orangutan females and social organization. In: Orangutans: Geographic variation in 
646

647

648

649

651

652

653

654

655

656

657

658

659

660

661

662

663

664

665

behavioral ecology and conservation $\left(\mathrm{ed}^{\wedge}(\mathrm{eds}\right.$ Wich SA, Utami-Atmoko SS, Mitra Setia T, van Schaik CP). Oxford University Press (2009).

56. Husson SJ, et al. Orangutan distribution, density, abundance and impacts of disturbance. In: Orangutans: Geographic variation in behavioral ecology and conservation ( $\mathrm{ed}^{\wedge}(\mathrm{eds}$ Wich SA, Utami-Atmoko SS, Mitra Setia T, van Schaik CP). Oxford University Press (2009).

57. Becker C. EEP studbook for zoo-housed orang-utans. (ed^(eds) (2016).

58. Friard O, Gamba M. BORIS: a free, versatile open-source event-logging software for video/audio coding and live observations. Methods in Ecology and Evolution 7, 1325-1330 (2016).

59. MacKinnon J. The behaviour and ecology of wild orang-utans (Pongo pygmaeus). Anim Behav 22, 3-74 (1974).

60. Rijksen HD. A fieldstudy on Sumatran orang utans (Pongo pygmaeus abelii, Lesson 1827): Ecology, behaviour and conservation. H. Veenman (1978).

61. Hardus M, et al. A description of the orangutan's vocal and sound repertoire, with a focus on geographical variation. In: Orangutans: Geographic variation in behavioral ecology and conservation $\left(\mathrm{ed}^{\wedge}(\mathrm{eds}\right.$ Wich SA, Utami-Atmoko SS, Mitra Setia T, van Schaik CP). Oxford University Press (2009).

62. Bakeman R, Quera V. Sequential analysis and observational methods for the behavioral sciences. Cambridge University Press (2011).

63. Baayen RH. Analyzing linguistic data. Cambridge University Press (2008).

64. Schielzeth $\mathrm{H}$, et al. Robustness of linear mixed $\square$ effects models to violations of distributional assumptions. Methods in Ecology and Evolution, (2020).

65. Knief U, Forstmeier W. Violating the normality assumption may be the lesser of two evils. bioRxiv, 498931 (2018).

66. Schielzeth H, Forstmeier W. Conclusions beyond support: overconfident estimates in mixed models. Behav Ecol 20, 416-420 (2009).

67. Pinheiro JC, Bates DM. Linear mixed-effects models: basic concepts and examples. Mixedeffects models in S and S-Plus, 3-56 (2000).

68. R Development Core Team. R: A language and environment for statistical computing. (ed^(eds). R Foundation for Statistical Computing (2017). 
666

667

668

669

670

671

672

673

674

675

676

677

678

679

680

681

682

683

684

685

686

687
69. Bates D, Maechler M, Bolker B, Walker S. lme4: Linear mixed-effects models using Eigen and S4. R package version 11-7, (2014).

70. Quinn GP, Keough MJ. Experimental design and data analysis for biologists. Cambridge University Press (2002).

71. Field A. Discovering statistics using SPSS. Sage publications (2005).

72. Fox J, Weisberg S. An R companion to applied regression, 2 edn. Sage (2011).

73. Forstmeier W, Schielzeth H. Cryptic multiple hypotheses testing in linear models: overestimated effect sizes and the winner's curse. Behav Ecol Sociobiol 65, 47-55 (2011).

74. Mundry R. Statistical Issues and Assumptions of Phylogenetic Generalized Least Squares (PGLS). In: Modern Phylogenetic Comparative Methods and Their Application in Evolutionary Biology (ed^(eds Garamszegi L). Springer (2014).

75. Dobson AJ. An Introduction to Generalized Linear Models. Chapman \& Hall/CRC (2002). 


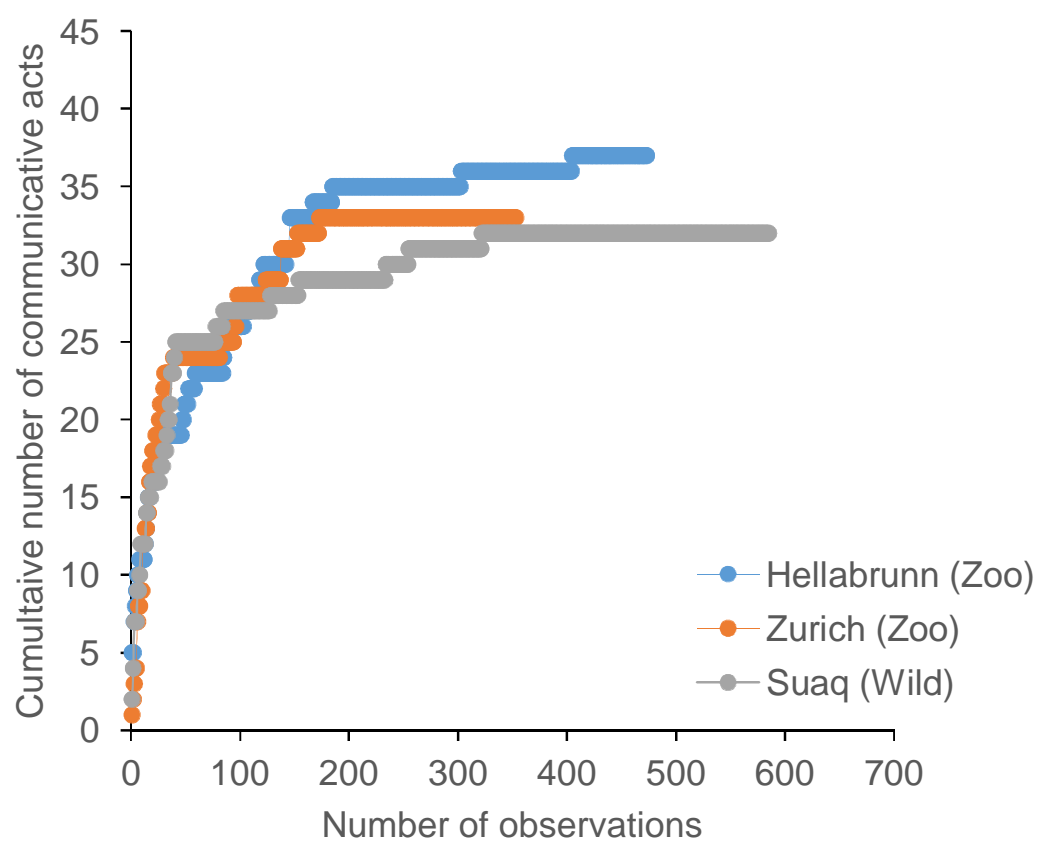
Sumatran orang-utans in this study.

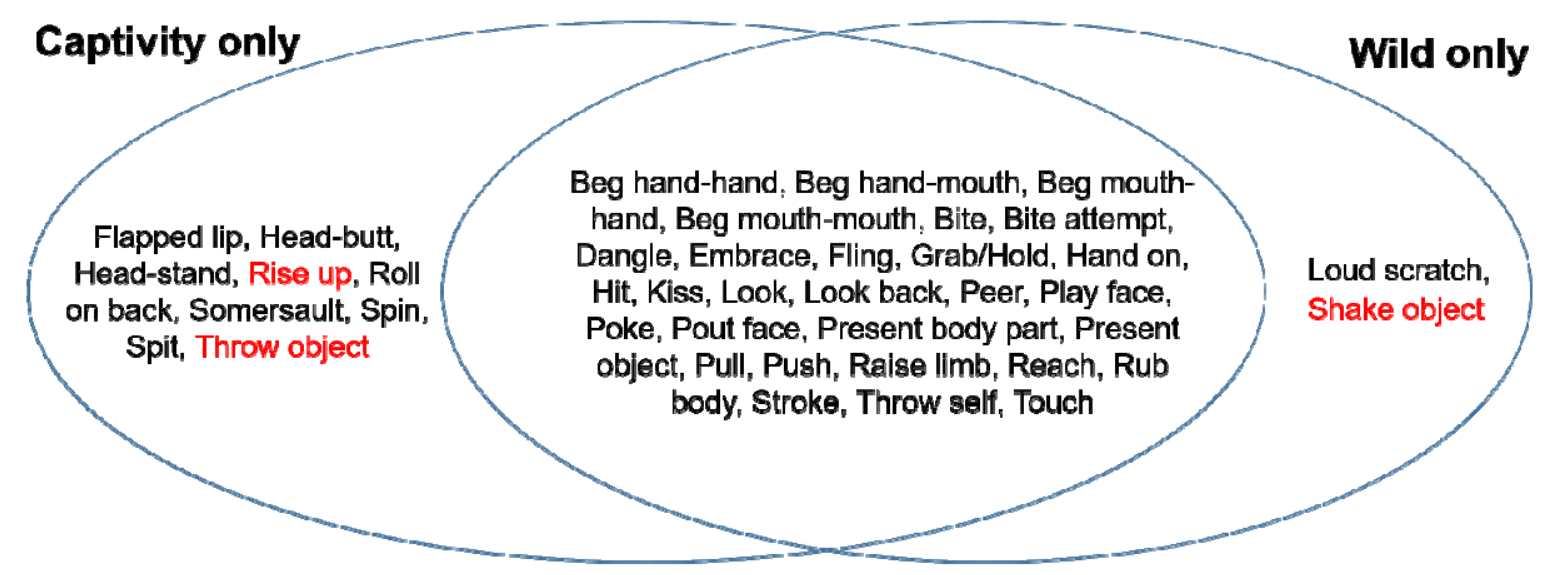

\section{All settings}

Fig. 2. Overview of communicative acts observed only in different research settings.

695 Communicative acts observed in the contrasting setting by other studies are marked in red. 
696
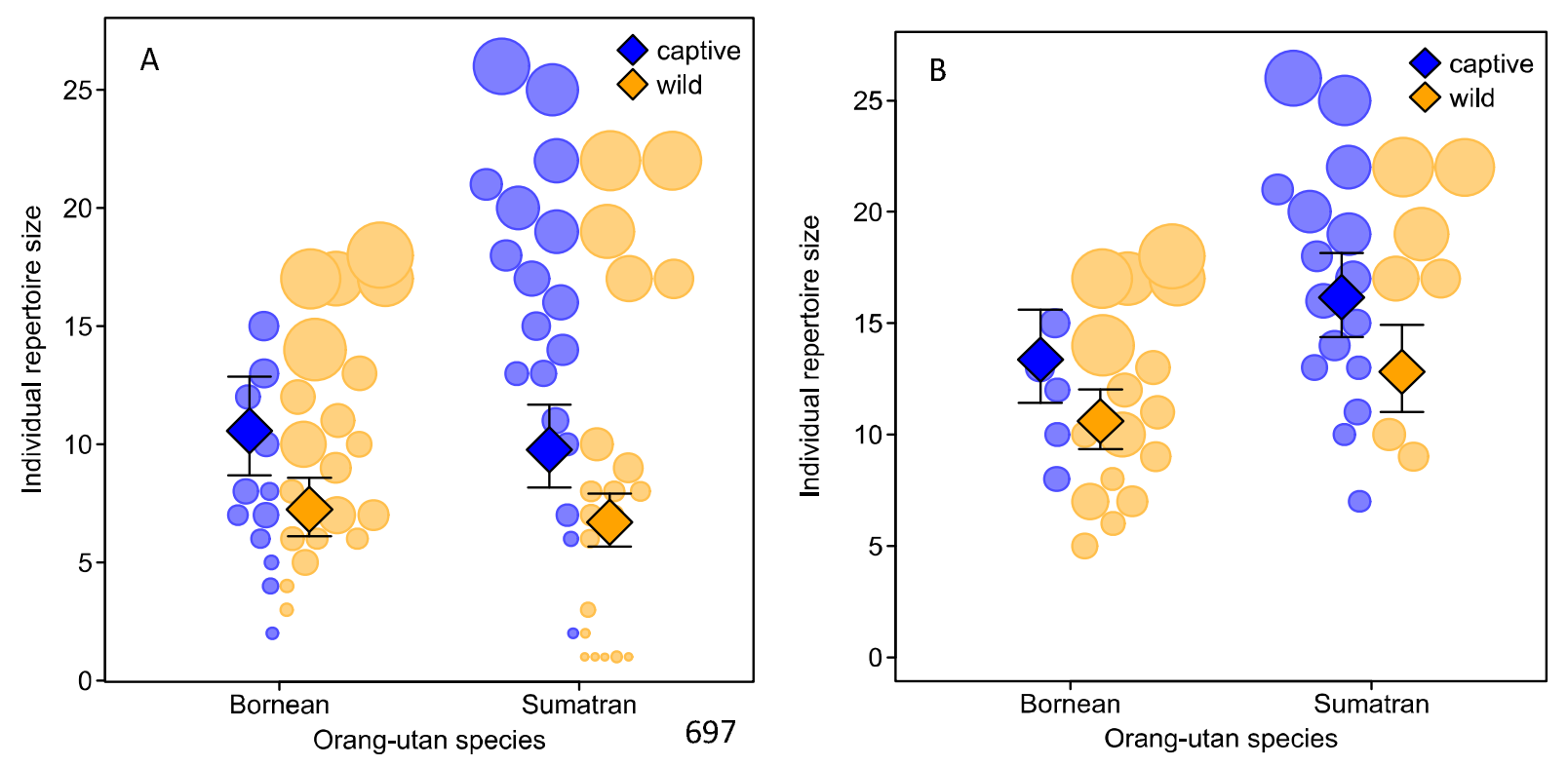

698 Fig. 3. Number of observed types of communicative acts per individual as a function of

699 research setting and orang-utan species, with (A) all subjects included, and (B) restricted to

700 subjects with $>50$ samples. Circles represent different individuals with area corresponding to sample

701 size, Diamonds depict model estimates with 95\% confidence intervals (all other variables centered to

702 a mean of zero). 


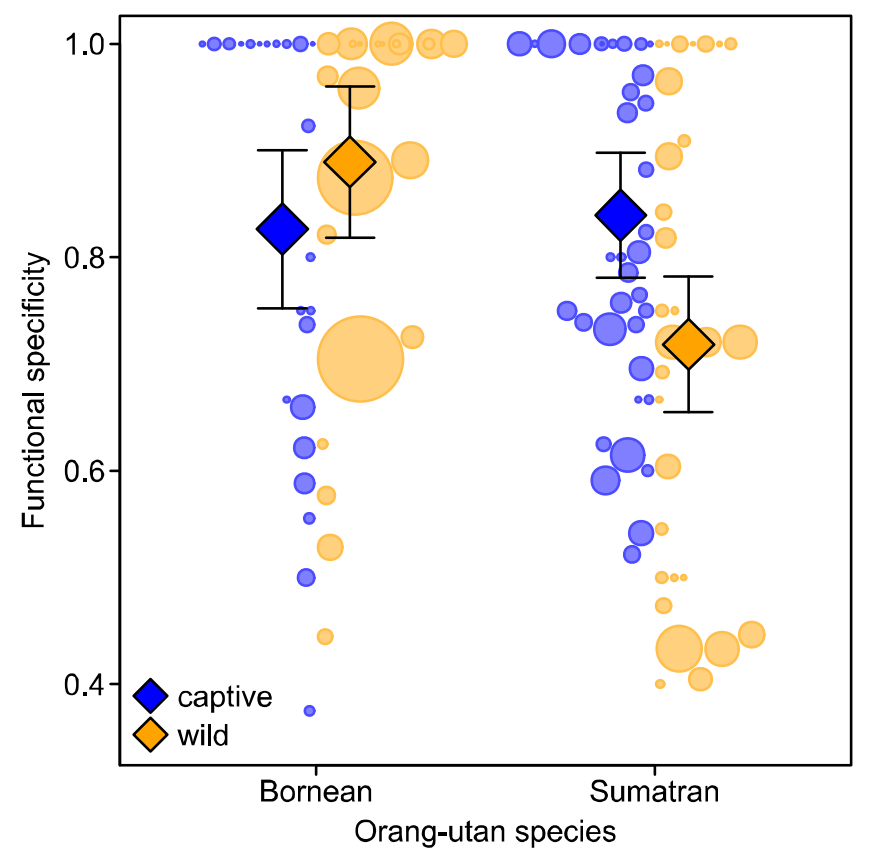

705 Fig. 4. Functional specificity of communicative acts as a function of research setting and orang-

706 utan species. Circles represent different signal types with area corresponding to sample size,

707 diamonds depict model estimates with $95 \%$ confidence intervals (all other variables centered to a 708 mean of zero). 
709 Tab. 1. Effects of research setting, orang-utan species, and control variables on (a) repertoire

710 size of individuals $(\mathrm{N}=44)$, and (b) functional specificity of communicative acts $(\mathrm{N}=114)$,

711 derived using LMMs with a Gaussian error structure and identity link function. Significant effects $(P$

$712<0.05)$ are depicted in italics.

\begin{tabular}{|c|c|c|c|c|}
\hline (a) Repertoire size & Estimate & $\mathrm{SE}$ & $\chi_{1}^{2}$ & $\mathrm{P}$ \\
\hline Intercept & 0.453 & 0.227 & - & - \\
\hline Setting [wild] & -0.29 & 0.069 & 12.727 & $<0.001$ \\
\hline Species [Sumatran] & 0.148 & 0.061 & 6.641 & 0.010 \\
\hline Age class [young imm.] & 0.314 & 0.076 & 14.571 & $<0.001$ \\
\hline Age class [old imm.] & 0.243 & 0.138 & 3.396 & 0.065 \\
\hline Sex [male] & -0.039 & 0.064 & 0.223 & 0.637 \\
\hline No. functions & 0.058 & 0.03 & 3.654 & 0.056 \\
\hline Log (no. observations) & 0.361 & 0.05 & 39.426 & $<0.001$ \\
\hline \multicolumn{5}{|l|}{ (b) Functional specificity } \\
\hline Intercept & 0.898 & 0.06 & - & - \\
\hline Setting [wild] & 0.059 & 0.043 & - & - \\
\hline Species [Sumatran] & 0.002 & 0.039 & - & - \\
\hline Dominant outcome [play] & 0.095 & 0.038 & 5.911 & 0.015 \\
\hline Log (no. observations) & 0.004 & 0.019 & 0.043 & 0.836 \\
\hline Log (no. subjects & -0.072 & 0.034 & 4.578 & 0.032 \\
\hline Setting $x$ species & -0.175 & 0.055 & 10.395 & 0.001 \\
\hline
\end{tabular}

